Verhaltenstherapie 1995;5:272

\title{
Subject Index Vol. 5, 1995
}

Agoraphobia 4200

Anxiety disorders 3 120,3 134

Behavioral disorders 4226

Blood pressure 4200

Cardiovascular deconditioning 4200

Case study 4217

Change of values 3149

Clinical assessment 142

Cognitive behaviour therapy 2 68, 296

disturbances 130

therapy 130 Comorbidity 3120 Conduct disorder 4226 Cortisol Suppl. 116 Daily hassles

Suppl. 116 Dental amalgam 4217 Diagnostic issues 3120 Disorder of sexual preference 3154

Eastern Germany 3149

Effects of psychotherapy 4232

Emotion and information processing 3138

Epidemiology 3120

Etiology 4207

Evaluation 3138

Experience sampling method Suppl. 116

Exposure in vivo 3134

treatment 3161 Expressed emotion Suppl. 11 Failure to thrive (MFT) 135 Feeding therapy 135

First-night effect 281 Fluoxetine 3154 Frotteurism 3154 Functional disorders Suppl. 19

Generalized anxiety disorder 4207 Health beliefs 4217

of female blue-collar workers 15

promotion 15

psychology 15 Heart rate 4200

Home-based self-help treatment 3134

Imipramine 3154

Insecurity 3149

Interventions 121

In vivo training 3154

Mental health 121

- $\quad$ retardation 135

Multimodal behavior therapy 3161

Obsessive compulsive disorder (OCD)

3161

Old age 121

Oppositional defiant disorder 4226

Organisational development 15 
Orthostatic intolerance 4200

Panic disorder 281

Paraphilia 3154

Personality development 15

Physical activity 121

Polysomnography 281

Posture 4200

Prevalence of mental disorders 3149

Prevention of marital distress Suppl. 11

Primary insomnia 281

Psychoeducational family therapy

Suppl. 11 Psychohygiene 3154 Psychophysiology Suppl. 19 Psychosocial relapse factors

Suppl. 11 Psychosomatic disorders 289

- $\quad$ symptoms 4217

Psychotherapy motivation 289

Quality assessment Suppl. 19

Relapse prevention 3154

Residential rehabilitation 296

-, vocational and recreational rehabilitation 268 Schizophrenia 1 30, 2 68, 2 96, Suppl. 11 Selfcontrol 3161 Self-experience 3 138,4 232 Self-management therapy 4232 Self-modification 4

232 Sexual delinquency 3154 Short-term group therapy 3134 Sleep 281 Social competence 2 96

- $\quad$ skills 268

Somatoform disorder 4217 Standardization 142 Stimulus control 3154 Stress prevention 15 - $\quad$ reactivity Suppl. 116

Suicide 3149

Test construction 289

Therapeutical competence 3138

Therapy 4207

Treatment planning 142

Video documentation/feedback 3161

Worries 4207

272 